\title{
Stability and Existence of Periodic Solutions for Cellular Neural Networks with State Dependent Delays on Time Scales
}

\author{
Yaping $\operatorname{Ren}^{1}$ and Yongkun $\mathrm{Li}^{2}$ \\ ${ }^{1}$ School of Statistics and Mathematics, Yunnan University of Finance and Economics, Yunnan, \\ Kunming 650221, China \\ ${ }^{2}$ Department of Mathematics, Yunnan University, Yunnan, Kunming 650091, China
}

Correspondence should be addressed to Yongkun Li, yklie@ynu.edu.cn

Received 12 April 2012; Accepted 27 August 2012

Academic Editor: Josef Diblík

Copyright (C) 2012 Y. Ren and Y. Li. This is an open access article distributed under the Creative Commons Attribution License, which permits unrestricted use, distribution, and reproduction in any medium, provided the original work is properly cited.

\begin{abstract}
We study delayed cellular neural networks on time scales. Without assuming the boundedness of the activation functions, we establish the exponential stability and existence of periodic solutions. The results in this paper are completely new even in case of the time scale $\mathbb{T}=\mathbb{R}$ or $\mathbb{Z}$ and improve some of the previously known results.
\end{abstract}

\section{Introduction}

Consider the following cellular neural networks with state-dependent delays on time scales:

$$
x_{i}^{\Delta}(t)=-c_{i}(t) x_{i}^{\sigma}(t)+\sum_{j=1}^{n} a_{i j}(t) f_{j}(x(t))+\sum_{j=1}^{n} b_{i j}(t) f_{j}\left(x_{j}\left(\xi_{i j}(t, x(t))\right)\right)+I_{i}(t), \quad t \in \mathbb{T},
$$

where $i=1,2, \ldots, n, \mathbb{T}$ is an $\omega$-periodic time scale which has the subspace topology inherited from the standard topology on $\mathbb{R}, x_{i}^{\sigma}(t)=x_{i}(\sigma(t)), \sigma(t)$ will be defined in the next section, $x(t)=\left(x_{1}(t), x_{2}(t), \ldots, x_{n}(t)\right), n$ corresponds to the number of units in the neural network, $x_{i}(t)$ corresponds to the state of the $i$ th unit at time $t, f_{j}\left(x_{j}(t)\right)$ denotes the output of the $j$ th unit on $i$ th unit at time $t, b_{i j}$ denotes the strength of the $j$ th unit on the $i$ th unit at time $\xi_{i j}(t, x(t)), I_{i}$ denotes the external bias on the $i$ th unit at time $t, t-\xi_{i j}(t, x(t))$ corresponds to the transmission delay along the axon of the $j$ th unit, $c_{i}$ represents the rate with which the $i$ th unit will reset its potential to the resting state in isolation when disconnected from the network and external inputs. 
It is well known that the cellular neural networks have been successfully applied to signal processing, pattern recognition, optimization, and associative memories, especially in image processing and solving nonlinear algebraic equations. They have been widely studied both in theory and applications [1-3]. Many results for the existence of their periodic solutions and the exponential convergence properties for cellular neural networks have been reported in the literatures. See, for instance, [4-17] and references cited therein.

In fact, continuous and discrete systems are very important in implementation and applications. It is well known that the theory of time scales has received a lot of attention which was introduced by Stefan Hilger in order to unify continuous and discrete analysis. Therefore, it is meaningful to study dynamic systems on time scales which can unify differential and difference systems see [18-28].

When $\mathbb{T}=\mathbb{R}, \xi_{i j}(t, \cdot) \equiv t-\tau_{i j}(t),(1.1)$ reduces to

$$
x_{i}^{\prime}(t)=-c_{i}(t) x_{i}(t)+\sum_{j=1}^{n} a_{i j}(t) f_{j}\left(x_{j}(t)\right)+\sum_{j=1}^{n} b_{i j}(t) f_{j}\left(x_{j}\left(t-\tau_{i j}(t)\right)\right)+I_{i}(t),
$$

where $i=1,2, \ldots, n$. By using Mawhin's continuation theorem and Liapunov functions, the authors $[6,14]$ obtained the existence and stability of periodic solutions of (1.2), respectively. see [15])

Furthermore, (1.1) also covers discrete system (for when $\mathbb{T}=\mathbb{Z}, \xi_{i j}(n, \cdot) \equiv n-\tau_{i j}(n)$;

$$
\begin{aligned}
x_{i}(n+1)= & x_{i}(n) e^{-c_{i}(n) h}+\theta_{i}(h) \sum_{j=1}^{m} a_{i j}(n) f_{j}\left(x_{j}(n)\right) \\
& +\theta_{i}(h) \sum_{j=1}^{m} b_{i j}(n) f_{j}\left(x_{j}\left(n-\tau_{i j}(n)\right)\right)+\theta_{i}(h) I_{i}(n),
\end{aligned}
$$

where $\theta_{i}(h)=\left(1-e^{-c_{i}(n) h}\right) / c_{i}(n), i=1,2, \ldots, m$. In [15], the author firstly obtained the discretetime analogue of (1.3) by the semidiscretization technique $[29,30]$, and then some sufficient conditions for the existence and global asymptotical stability of periodic solutions of (1.3) were established by using Mawhin's continuation theorem and Liapunov functions.

However, in $[5,13-15]$, the activation functions $f_{j}, j=1,2, \ldots, n$ are assumed to be bounded. Our main purpose of this paper is to establish the stability and existence of periodic solutions of (1.1) without assuming the boundedness of the activation functions.

For the sake of convenience, we denote

$$
\begin{array}{cc}
\bar{c}_{i}=\max _{t \in[0, \omega]_{\mathbb{T}}}\left|c_{i}(t)\right|, \quad \underline{c}_{i}=\min _{t \in[0, \omega]_{\mathbb{T}}}\left|c_{i}(t)\right|, \quad \bar{a}_{i j}=\max _{t \in[0, \omega]_{\mathbb{T}}}\left|a_{i j}(t)\right|, \\
\bar{b}_{i j}=\max _{t \in[0, \omega]_{\mathbb{T}}}\left|b_{i j}(t)\right|, \quad \bar{I}_{i}=\max _{t \in[0, \omega]_{\mathbb{T}}}\left|I_{i}(t)\right|, \quad i, j=1,2, \ldots, n .
\end{array}
$$

Throughout this paper, we assume that

(H1) $\xi_{i j}(\cdot, \cdot) \in C\left(\mathbb{T} \times \mathbb{R}^{n},[0, \infty)_{\mathbb{T}}\right)$ is $\omega$-periodic with respect to its first argument and satisfies $\xi_{i j}(t, x) \leq \tau$ for all $(t, x) \in \mathbb{T} \times \mathbb{R}^{n}, c_{i} \in C(\mathbb{T},(0,+\infty)), a_{i j}, b_{i j}, I_{i} \in C(\mathbb{T}, \mathbb{R}), i$, $j=1,2, \ldots, n$ are $\omega$-periodic functions; 
(H2) $f_{j} \in C(\mathbb{R}, \mathbb{R})$, and there exists a positive number $M_{j}$ such that $\left|f_{j}(x)-f_{j}(y)\right| \leqslant$ $M_{j}|x-y|$ for all $x, y \in \mathbb{R}, j=1,2, \ldots, n$.

The initial conditions of system (1.1) are of the following form:

$$
x_{i}(s)=\phi_{i}(s), \quad i=1,2, \ldots, n, s \in[-\tau, 0]_{\mathbb{T}}, \quad \tau=\max _{1 \leqslant i \leqslant n, 1 \leqslant j \leqslant n \in[0, \omega]_{\mathbb{T}}}\left|\tau_{i j}(t)\right|,
$$

where $\phi_{i} \in C\left([-\tau, 0]_{\mathbb{T}}, \mathbb{R}\right), i=1,2, \ldots, n$.

The organization of this paper is as follows. In Section 2, we introduce some lemmas and definitions and state some preliminary results needed in later sections, which will be used in latter sections. In Section 3, we will study the existence of periodic solutions of system (1.1) by using the method of coincidence degree. In Section 4, we will derive sufficient conditions to ensure that the periodic solutions of (1.1) are globally exponentially stable. In Section 5, an example is also provided to illustrate the effectiveness of the main results in Sections 3 and 4. The conclusions are drawn in Section 6.

\section{Preliminaries}

In this section, we will introduce some notations and definitions and state some preliminary results.

Let $\mathbb{T}$ be a nonempty closed subset (time scale) of $\mathbb{R}$. The forward and backward jump operators $\sigma, \rho: \mathbb{T} \rightarrow \mathbb{T}$ and the graininess $\mu: \mathbb{T} \rightarrow \mathbb{R}^{+}$are defined, respectively, by

$$
\sigma(t)=\inf \{s \in \mathbb{T}: s>t\}, \quad \rho(t)=\sup \{s \in \mathbb{T}: s<t\}, \quad \mu(t)=\sigma(t)-t .
$$

A point $t \in \mathbb{T}$ is called left dense if $t>\inf \mathbb{T}$ and $\rho(t)=t$, left scattered if $\rho(t)<t$, right dense if $t<\sup \mathbb{T}$ and $\sigma(t)=t$, and right scattered if $\sigma(t)>t$. If $\mathbb{T}$ has a left-scattered maximum $m$, then $\mathbb{T}^{k}=\mathbb{T} \backslash\{m\}$; otherwise $\mathbb{T}^{k}=\mathbb{T}$. If $\mathbb{T}$ has a right-scattered minimum $m$, then $\mathbb{T}_{k}=\mathbb{T} \backslash\{m\}$; otherwise $\mathbb{T}_{k}=\mathbb{T}$.

A function $f: \mathbb{T} \rightarrow \mathbb{R}$ is right dense continuous provided that it is continuous at rightdense point in $\mathbb{T}$, and its left-side limits exist at left-dense points in $\mathbb{T}$. If $f$ is continuous at each right-dense point and each left-dense point, then $f$ is said to be continuous function on $\mathbb{T}$. The set of continuous functions $f: \mathbb{T} \rightarrow \mathbb{R}$ will be denoted by $C(\mathbb{T})$.

For $y: \mathbb{T} \rightarrow \mathbb{R}$ and $t \in \mathbb{T}^{k}$, we define the delta derivative of $y(t), y^{\Delta}(t)$, to be the number (if it exists) with the property that for a given $\varepsilon>0$, there exists a neighborhood $U$ of $t$ such that

$$
\left|[y(\sigma(t))-y(s)]-y^{\Delta}(t)[\sigma(t)-s]\right|<\varepsilon|\sigma(t)-s| \quad \forall s \in U
$$

If $y$ is continuous, then $y$ is right-dense continuous, and if $y$ is delta differentiable at $t$, then $y$ is continuous at $t$.

Let $y$ be right dense continuous. If $Y^{\Delta}(t)=y(t)$, then we define the delta integral by $\int_{a}^{t} y(s) \Delta s=Y(t)-Y(a)$.

Definition 2.1 (see [31]). We say that a time scale $\mathbb{T}$ is periodic if there exists $p>0$ such that if $t \in \mathbb{T}$, then $t \pm p \in \mathbb{T}$. For $\mathbb{T} \neq \mathbb{R}$, the smallest positive $p$ is called the period of the time scale. 
Definition 2.2 (see [31]). Let $\mathbb{T} \neq \mathbb{R}$ be a periodic time scale with period $p$. We say that the function $f: \mathbb{T} \rightarrow \mathbb{R}$ is periodic with period $\omega$ if there exists a natural number $n$ such that $\omega=n p, f(t+\omega)=f(t)$ for all $t \in \mathbb{T}$, and $\omega$ is the smallest number such that $f(t+\omega)=f(t)$. If $\mathbb{T}=\mathbb{R}$, we say that $f$ is periodic with period $\omega>0$ if $\omega$ is the smallest positive number such that $f(t+\omega)=f(t)$ for all $t \in \mathbb{T}$.

If $\mathbb{T}$ is $\omega$ periodic, then $\sigma(t+\omega)=\sigma(t)+\omega$ and $\mu(t)$ is an $\omega$-periodic function.

Definition 2.3 (see [32]). A function $p: \mathbb{T} \rightarrow \mathbb{R}$ is said to be regressive provided that $1+$ $\mu(t) p(t) \neq 0$ for all $t \in \mathbb{T}^{k}$, where $\mu(t)=\sigma(t)-t$ is the graininess function. The set of all regressive rd-continuous functions $f: \mathbb{T} \rightarrow \mathbb{R}$ is denoted by $R$ while the set $R^{+}$is given by $\{f \in \mathcal{R}: 1+\mu(t) f(t)>0\}$ for all $t \in \mathbb{T}$. Let $p \in \mathcal{R}$. The exponential function is defined by

$$
e_{p}(t, s)=\exp \left(\int_{s}^{t} \xi_{\mu(\tau)}(p(\tau)) \Delta \tau\right)
$$

where $\xi_{h(z)}$ is the so-called cylinder transformation.

Let $p, q: \mathbb{T} \rightarrow \mathbb{R}$ be two regressive functions, and we define

$$
p \oplus q:=p+q+\mu p q, \quad \ominus p:=-\frac{p}{1+\mu p}, \quad p \ominus q:=p \oplus(\ominus q)
$$

Then the generalized exponential function has the following properties.

Lemma 2.4 (see [32]). Assume that $p, q: \mathbb{T} \rightarrow \mathbb{R}$ are two regressive functions, and then

(1) $e_{p}(\sigma(t), s)=(1+\mu(t) p(t)) e_{p}(t, s)$;

(2) $e_{p}(t, s)=1 / e_{p}(s, t)=e_{\ominus p}(s, t)$;

(3) $e_{p}(t, s) e_{p}(s, r)=e_{p}(t, r)$.

Lemma 2.5 (see [32]). Assume that $f, g: \mathbb{T} \rightarrow \mathbb{R}$ are delta differentiable at $t \in \mathbb{T}^{k}$. Then

$$
(f g)^{\Delta}(t)=f^{\Delta}(t) g(t)+f(\sigma(t)) g^{\Delta}(t)=f(t) g^{\Delta}(t)+f^{\Delta}(t) g(\sigma(t)) .
$$

Lemma 2.6 (see [32]). If $a, b \in \mathbb{T}, \alpha, \beta \in \mathbb{R}$, and $f, g \in C(\mathbb{T}, \mathbb{R})$, then

(1) $\int_{a}^{b}[\alpha f(t)+\beta g(t)] \Delta t=\alpha \int_{a}^{b} f(t) \Delta t+\alpha \int_{a}^{b} g(t) \Delta t$;

(2) if $f(t) \geq 0$ for all $a \leq t<b$, then $\int_{a}^{b} f(t) \Delta t \geq 0$;

(3) if $|f(t)| \leq g(t)$ on $[a, b):=\{t \in \mathbb{T}: a \leq t<b\}$, then $\left|\int_{a}^{b} f(t) \Delta t\right| \leq \int_{a}^{b} g(t) \Delta t$.

Lemma 2.7 (see [33]). Let $t_{1}, t_{2} \in[0, \omega]_{\mathbb{T}}$. If $x: \mathbb{T} \rightarrow \mathbb{R}$ is $\omega$ periodic, then

$$
x(t) \leqslant x\left(t_{1}\right)+\int_{0}^{\omega}\left|x^{\Delta}(s)\right| \Delta s, \quad x(t) \geqslant x\left(t_{2}\right)-\int_{0}^{\omega}\left|x^{\Delta}(s)\right| \Delta s .
$$


Definition 2.8. The periodic solution $x^{*}(t)=\left(x_{1}^{*}(t), x_{2}^{*}(t), \ldots, x_{n}^{*}(t)\right)^{T}$ of system (1.1) is said to be exponentially stable if there exists a positive constant $\alpha$ with $\ominus \alpha \in \mathcal{R}^{+}$such that for every $\delta \in[-\tau, 0]_{\mathbb{T}}$, and there exists $N=N(\delta) \geqslant 1$ such that the solution $x(t)=$ $\left(x_{1}(t), x_{2}(t), \ldots, x_{n}(t)\right)^{T}$ of system (1.1) through $(\delta, x(\delta))$ satisfies

$$
\sum_{i=1}^{n}\left|x_{i}(t)-x_{i}^{*}(t)\right| \leqslant N\left\|\phi(\delta)-x^{*}(\delta)\right\| e_{\ominus \alpha}(t, \delta), \quad t \in \mathbb{T}^{+}:=[0, \infty)_{\mathbb{T}}
$$

where $\left\|\phi(\delta)-x^{*}(\delta)\right\|=\sum_{i=1}^{n} \max _{\delta \in[-\tau, 0]_{\mathbb{T}}}\left|\phi_{i}(\delta)-x_{i}^{*}(\delta)\right|$.

In order to show that there exists at least one $\omega$-periodic solution of system (1.1), we need the following concepts and result which are cited from [34].

Let $\mathbb{X}, \mathbb{Y}$ be Banach spaces, $L:$ Dom $L \subset \mathbb{X} \rightarrow \operatorname{dim} \mathbb{Y}$ be a linear mapping, and $N$ : $\mathbb{X} \rightarrow \mathbb{Y}$ be a continuous mapping. The mapping $L$ will be called a Fredholm mapping of index zero if $\operatorname{dim} \operatorname{Ker} L=$ co dim $\operatorname{Im} L<+\infty$ and $\operatorname{Im} L$ is closed in $\mathbb{Y}$. If $L$ is a Fredholm mapping of index zero and there exists continuous projector $P: \mathbb{X} \rightarrow \mathbb{X}$ and $Q: \mathbb{Y} \rightarrow \mathbb{Y}$ such that $\operatorname{Im} P=\operatorname{Ker} L, \operatorname{Ker} Q=\operatorname{Im}(I-Q)$, it follows that mapping $\left.L\right|_{\text {Dom } L \cap K e r} P:(I-P) \mathbb{X} \rightarrow \operatorname{Im} L$ is invertible. We denote the inverse of that mapping by $K_{P}$. If $\Omega$ is an open bounded subset of $\mathbb{X}$, the mapping $N$ will be called $L$ compact on $\bar{\Omega}$ if $Q N(\bar{\Omega})$ is bounded and $K_{P}(I-Q) N: \bar{\Omega} \rightarrow \mathbb{X}$ is compact.

Lemma 2.9 (see [34]). Let $\mathbb{X}, \mathbb{Y}$ be two Banach spaces and $\Omega \subset \mathbb{X}$ be open bounded and symmetric with $0 \in \Omega$. Suppose that $L: D(L) \subset \mathbb{X} \rightarrow \mathbb{Y}$ is a linear Fredholm operator of index zero with $D(L) \cap \bar{\Omega} \neq \emptyset$, and $N: \bar{\Omega} \rightarrow \mathbb{Y}$ is $L$ compact. Further, one also assumes that

(H) $L x-N x \neq \lambda(-L x-N(-x))$ for all $x \in D(L) \cap \partial \Omega, \lambda \in(0,1]$.

Then equation $L x=N x$ has at least one solution on $D(L) \cap \bar{\Omega}$.

Definition 2.10 (see [35]). A real matrix $P=\left(p_{i j}\right)_{n \times n}$ is said to be a nonsingular $M$ matrix if $p_{i j} \leq 0, i, j=1,2, \ldots, n, i \neq j$, and all successive principal minors of $P$ are positive.

For $A=\left(a_{i j}\right)_{m \times n}, B=\left(b_{i j}\right)_{m \times n} \in \mathbb{R}^{m n}, A \geq B(A>B)$ means that each pair of corresponding elements of $A$ and $B$ such that $a_{i j} \geq b_{i j}\left(a_{i j}>b_{i j}\right)$.

Lemma 2.11 (see [35]). Assume that $P$ is a nonsingular $M$ matrix and $P y \leq h$, then $y \leq P^{-1} h$.

\section{Existence of Periodic Solutions}

In this section, by Lemma 2.9, we will study the existence of at least one periodic solution of system (1.1).

Theorem 3.1. Suppose that (H1)-(H2) holds, $E=\left(e_{i j}\right)_{n \times n}$ is a nonsingular M matrix, where

$$
e_{i j}=z \begin{cases}r_{i}+d_{i i} & i=j, \\ d_{i j} & i \neq j,\end{cases}
$$


and $r_{i}=\underline{c}_{i}\left(1-\omega \bar{c}_{i}\right) /\left(1+\underline{c}_{i} \omega\right), d_{i j}=-\left(\bar{a}_{i j}+\bar{b}_{i j}\right) M_{j}, i, j=1,2, \ldots, n$, then system (1.1) has at least one $\omega$-periodic solution.

Proof. Let

$$
\mathbb{X}=\mathbb{Y}=\left\{x=\left(x_{1}, x_{2}, \ldots, x_{n}\right)^{T} \in C(\mathbb{T}, \mathbb{R}): x(t+\omega)=x(t), t \in \mathbb{T}\right\}
$$

with the norm defined by $\|x\|=\max _{1 \leq i \leq n}\left|x_{i}\right|_{0}$, where $\left|x_{i}\right|_{0}=\max _{t \in[0, \omega]_{\mathbb{T}}}\left|x_{i}(t)\right|$, then $\mathbb{X}$ and $\mathbb{Y}$ are Banach spaces.

Set

$$
L x=x^{\Delta}(t), \quad P x=Q x=\frac{1}{\omega} \int_{0}^{\omega} x(t) \Delta t, \quad x \in \mathbb{X}
$$

and $\mathbb{N}: \mathbb{X} \rightarrow \mathbb{Y}$

$$
N x_{i}(t)=-c_{i}(t) x_{i}^{\sigma}(t)+\sum_{j=1}^{n} a_{i j}(t) f_{j}\left(x_{j}(t)\right)+\sum_{j=1}^{n} b_{i j}(t) f_{j}\left(x_{j}\left(\xi_{i j}(t, x(t))\right)\right)+I_{i}(t),
$$

where $i=1,2, \ldots, n$. Obviously, $\operatorname{Ker} L=\mathbb{R}^{n}, \operatorname{Im} L=\left\{x \in \mathbb{X}: \int_{0}^{\omega} x(s) \Delta s=0\right\}$ is closed in $\mathbb{Y}$ and

$\operatorname{dim} \operatorname{Ker} L=n=\operatorname{codim} \operatorname{Im} L$.

Hence, $L$ is a Fredholm mapping of index zero. Furthermore, similar to the proof of Theorem 3.4 in [21], one can easily show that $N$ is $L$ compact on $\bar{\Omega}$ with any open bounded set $\Omega \subset X$. Corresponding to the operator equation $L x-N x=\lambda(-L x-N(-x)), \lambda \in(0,1]$, we have

$$
\begin{aligned}
x_{i}^{\Delta}(t)= & \frac{1}{1+\lambda}\left[-c_{i}(t) x_{i}^{\sigma}(t)+\sum_{j=1}^{n} a_{i j}(t) f_{j}\left(x_{j}(t)\right)+\sum_{j=1}^{n} b_{i j}(t) f_{j}\left(x_{j}\left(\xi_{i j}(t, x(t))\right)\right)+I_{i}(t)\right] \\
& -\frac{\lambda}{1+\lambda}\left[c_{i}(t) x_{i}^{\sigma}(t)+\sum_{j=1}^{n} a_{i j}(t) f_{j}\left(-x_{j}(t)\right)+\sum_{j=1}^{n} b_{i j}(t) f_{j}\left(-x_{j}\left(\xi_{i j}(t, x(t))\right)\right)+I_{i}(t)\right],
\end{aligned}
$$

where $i=1,2, \ldots, n$. 
Suppose that $x=\left(x_{1}, x_{2}, \ldots, x_{n}\right)^{T}$ is a solution of system (3.6) for some $\lambda \in(0,1]$. In view of (3.6) and (H2), we have

$$
\begin{aligned}
\int_{0}^{\omega}\left|x_{i}^{\Delta}(t)\right| \Delta t \leq & \int_{0}^{\omega}\left|c_{i}(t) x_{i}^{\sigma}(t)\right| \Delta t+\int_{0}^{\omega}\left|I_{i}(t)\right| \Delta t \\
& +\frac{1}{1+\lambda} \int_{0}^{\omega}\left|\left[\sum_{j=1}^{n} \bar{a}_{i j} f_{j}\left(x_{j}(t)\right)+\sum_{j=1}^{n} \bar{b}_{i j} f_{j}\left(x_{j}\left(\xi_{i j}(t, x(t))\right)\right)\right]\right| \Delta t \\
& +\frac{\lambda}{1+\lambda} \int_{0}^{\omega}\left|\left[\sum_{j=1}^{n} \bar{a}_{i j} f_{j}\left(-x_{j}(t)\right)+\sum_{j=1}^{n} \bar{b}_{i j} f_{j}\left(-x_{j}\left(\xi_{i j}(t, x(t))\right)\right)\right]\right| \Delta t \\
\leqslant & \int_{0}^{\omega} \bar{c}_{i}\left|x_{i}^{\sigma}(t)\right| \Delta t+\int_{0}^{\omega}\left[\sum_{j=1}^{n} \bar{a}_{i j}\left(M_{j}\left|x_{j}(t)\right|+\left|f_{j}(0)\right|\right)\right. \\
\leqslant & \bar{c}_{i} \omega\left|x_{i}\right|_{0}+\omega \sum_{j=1}^{n}\left(\bar{a}_{i j}+\bar{b}_{i j}\right) M_{j}\left|x_{j}\right|_{0}+\omega\left[\sum_{j=1}^{n}\left(\bar{a}_{i j}+\bar{b}_{i j}\right)\left|f_{j}(0)\right|+\bar{I}_{i}\right] \\
\leqslant & \left.\left.\left.\left.\bar{c}_{i} \omega\left|x_{i}\right|_{0}+\omega \sum_{j=1}^{n}\left(\bar{a}_{i j}+\bar{b}_{i j}\right) M_{j}\left|x_{j}\right|_{0}+\omega(t)\right)\right)|+| x_{j}(0) \mid\right)+\bar{I}_{i}\right] \Delta t
\end{aligned}
$$

where $\alpha_{i}=\sum_{j=1}^{n}\left(\bar{a}_{i j}+\bar{b}_{i j}\right)\left|f_{j}(0)\right|+\bar{I}_{i}$.

Integrating both sides of (3.6) from 0 to $\omega$, we obtain that

$$
\begin{aligned}
\int_{0}^{\omega} c_{i}(t) x_{i}^{\sigma}(t) \Delta t= & \frac{1}{1+\lambda} \int_{0}^{\omega}\left[\sum_{j=1}^{n} a_{i j}(t) f_{j}\left(x_{j}(t)\right)+\sum_{j=1}^{n} b_{i j}(t) f_{j}\left(x_{j}\left(\xi_{i j}(t, x(t))\right)\right)+I_{i}(t)\right] \Delta t \\
& -\frac{\lambda}{1+\lambda} \int_{0}^{\omega}\left[\sum_{j=1}^{n} a_{i j}(t) f_{j}\left(-x_{j}(t)\right)+\sum_{j=1}^{n} b_{i j}(t) f_{j}\left(-x_{j}\left(\xi_{i j}(t, x(t))\right)\right)+I_{i}(t)\right] \Delta t,
\end{aligned}
$$

where $i=1,2, \ldots, n$. Then we have from (3.7) that

$$
\begin{aligned}
\left|\int_{0}^{\omega} c_{i}(t) x_{i}^{\sigma}(t) \Delta t\right| & \leqslant \int_{0}^{\omega}\left[\sum_{j=1}^{n}\left|a_{i j}(t) f_{j}\left(x_{j}(t)\right)\right|+\sum_{j=1}^{n}\left|b_{i j}(t) f_{j}\left(x_{j}\left(\xi_{i j}(t, x(t))\right)\right)\right|+\left|I_{i}(t)\right|\right] \Delta t \\
& \leqslant \omega \sum_{j=1}^{n}\left(\bar{a}_{i j}+\bar{b}_{i j}\right) M_{j}\left|x_{j}\right|_{0}+\omega \alpha_{i}, \quad i=1,2, \ldots, n .
\end{aligned}
$$


From Lemma 2.7 , for any $t_{1}^{i}, t_{2}^{i} \in[0, \omega]_{\mathbb{T}}, i=1,2, \ldots, n$, we have

$$
\begin{gathered}
\int_{0}^{\omega} c_{i}(t) x_{i}^{\sigma}(t) \Delta t \leqslant \int_{0}^{\omega} c_{i}(t) x_{i}\left(t_{1}^{i}\right) \Delta t+\int_{0}^{\omega} c_{i}(t)\left(\int_{0}^{\omega}\left|x_{i}^{\Delta}(t)\right| \Delta t\right) \Delta t, \\
\int_{0}^{\omega} c_{i}(t) x_{i}^{\sigma}(t) \Delta t \geqslant \int_{0}^{\omega} c_{i}(t) x_{i}\left(t_{2}^{i}\right) \Delta t-\int_{0}^{\omega} c_{i}(t)\left(\int_{0}^{\omega}\left|x_{i}^{\Delta}(t)\right| \Delta t\right) \Delta t, \quad i=1,2, \ldots, n .
\end{gathered}
$$

Dividing by $\int_{0}^{\omega} c_{i}(t) \Delta t$ on the two sides of the inequalities above, we obtain that

$$
\begin{gathered}
-x_{i}\left(t_{1}^{i}\right) \leqslant-\frac{1}{\int_{0}^{\omega} c_{i}(t) \Delta t} \int_{0}^{\omega} c_{i}(t) x_{i}^{\sigma}(t) \Delta t+\int_{0}^{\omega}\left|x_{i}^{\Delta}(t)\right| \Delta t, \\
x_{i}\left(t_{2}^{i}\right) \leqslant \frac{1}{\int_{0}^{\omega} c_{i}(t) \Delta t} \int_{0}^{\omega} c_{i}(t) x_{i}^{\sigma}(t) \Delta t+\int_{0}^{\omega}\left|x_{i}^{\Delta}(t)\right| \Delta t, \quad i=1,2, \ldots, n .
\end{gathered}
$$

Let $\left|x_{i}\left(t_{0}^{i}\right)\right|=\max _{t \in[0, \omega]_{T}}\left|x_{i}(t)\right|, i=1,2, \ldots, n$. If for some $i=1,2, \ldots, n, x_{i}\left(t_{0}^{i}\right) \geqslant 0$, we choose $t_{2}^{i}=t_{0}^{i}$. Hence $\max _{t \in[0, \omega]_{\mathbb{T}}}\left|x_{i}(t)\right|=x_{i}\left(t_{2}^{i}\right)$. From (3.12), we have

$$
\begin{aligned}
\underline{c}_{i}\left|x_{i}\right|_{0} & \leqslant \underline{c}_{i}\left(\frac{1}{\int_{0}^{\omega} c_{i}(t) \Delta t} \int_{0}^{\omega} c_{i}(t) x_{i}^{\sigma}(t) \Delta t+\int_{0}^{\omega}\left|x_{i}^{\Delta}(t)\right| \Delta t\right) \\
& \leqslant \frac{1}{\omega}\left|\int_{0}^{\omega} c_{i}(t) x_{i}^{\sigma}(t) \Delta t\right|+\underline{c}_{i} \int_{0}^{\omega}\left|x_{i}^{\Delta}(t)\right| \Delta t .
\end{aligned}
$$

If for some $i=1,2, \ldots, n, x_{i}\left(t_{0}^{i}\right) \leqslant 0$, we choose $t_{1}^{i}=t_{0}^{i}$. Hence $\max _{t \in[0, \omega]_{\mathbb{T}}}\left|x_{i}(t)\right|=-x_{i}\left(t_{1}^{i}\right)$. From (3.11), we also have (3.13).

By using (3.7) and (3.9) and (3.13), for $i=1,2, \ldots, n$, we obtain that

$$
\underline{c}_{i}\left|x_{i}\right|_{0} \leqslant \sum_{j=1}^{n}\left(\bar{a}_{i j}+\bar{b}_{i j}\right) M_{j}\left|x_{j}\right|_{0}+\alpha_{i}+\bar{c}_{i} \omega\left|x_{i}\right|_{0}+\underline{c}_{i} \omega \sum_{j=1}^{n}\left(\bar{a}_{i j}+\bar{b}_{i j}\right) M_{j}\left|x_{j}\right|_{0}+\underline{c}_{i} \omega \alpha_{i},
$$

so,

$$
\frac{\underline{c}_{i}\left(1-\omega \bar{c}_{i}\right)}{\left(1+\underline{c}_{i} \omega\right)}\left|x_{i}\right|_{0} \leqslant \sum_{j=1}^{n}\left(\bar{a}_{i j}+\bar{b}_{i j}\right) M_{j}\left|x_{j}\right|_{0}+\alpha_{i},
$$

namely,

$$
r_{i}\left|x_{i}\right|_{0}-\sum_{j=1}^{n} d_{i j}\left|x_{j}\right|_{0} \leqslant \alpha_{i}, \quad i=1,2, \ldots, n
$$

Denote the following:

$$
|x|_{0}=\left(\left|x_{1}\right|_{0},\left|x_{2}\right|_{0}, \ldots,\left|x_{n}\right|_{0}\right)^{T}, \quad C=\left(\alpha_{1}, \alpha_{2}, \ldots, \alpha_{n}\right)^{T} .
$$


Thus (3.16) is rewritten in the matrix form

$$
E|x|_{0} \leqslant C
$$

From the conditions of Theorem 3.1, $E$ is a nonsingular $M$ matrix, therefore

$$
|x|_{0} \leqslant E^{-1} C:=\left(B_{1}, B_{2}, \ldots, B_{n}\right)^{T},
$$

that is, $\left|x_{i}\right|_{0} \leqslant B_{i}, i=1,2, \ldots, n$.

Take $A=\max _{1 \leq i \leq n} B_{i}+1$ and

$$
\Omega=\{x \in \mathbb{X}:\|x\|<A\}
$$

It is clear that $\Omega$ satisfies all the requirements in Lemma 2.9 , and condition $(\mathrm{H})$ is satisfied. In view of all the discussions above, we conclude from Lemma 2.9 that system (1.1) has at least one $\omega$-periodic solution. This completes the proof.

From Theorem 3.1, when $\mathbb{T}=\mathbb{R}, \xi_{i j}(t, \cdot) \equiv t-\tau_{i j}(t)$, we have the following.

Corollary 3.2. Under assumptions of Theorem 3.1, system (1.2) has at least one w-periodic solution.

When $\mathbb{T}=\mathbb{Z}, \xi_{i j}(n, \cdot) \equiv n-\tau_{i j}(n)$, from Theorem 3.1, we have the following.

Corollary 3.3. Under assumptions of Theorem 3.1, system (1.3) has at least one w-periodic solution.

\section{Global Exponential Stability of the Periodic Solution}

In this section, we will establish some results for the global exponential stability of the periodic solution of (1.1). The following lemma is essential.

Lemma 4.1 (see [36]). Let $p: \mathbb{T} \rightarrow \mathbb{R}$ be rd-continuous and regressive. Suppose that $f: \mathbb{T} \rightarrow \mathbb{R}$ is $r d$-continuous, $\delta \in \mathbb{T}$, and $y_{0} \in \mathbb{R}$. Then $y$ is the unique solution of the initial value problem

$$
y^{\Delta}(t)+p(t) y^{\sigma}(t)=h(t), \quad y(\delta)=y_{0}
$$

if and only if

$$
y(t)=e_{\ominus p}(t, \delta) y_{0}+\int_{\delta}^{t} e_{\ominus p}(t, s) h(s) \Delta s
$$

Theorem 4.2. Assume $\left(H_{1}\right),\left(H_{2}\right)$, and $\left(H_{4}\right)$ hold. Furthermore, suppose that

$\left(\mathrm{H}_{5}\right)$ for $t \in[0, \omega]_{\mathbb{T}}, i=1,2, \ldots, n, c_{i}(t)-\widehat{M}>0$, where $\widehat{M}=\max _{1 \leq i \leq n}\left\{\sum_{j=1}^{n} \bar{a}_{i j} M_{j}+\right.$ $\left.\sum_{j=1}^{n} \bar{b}_{i j} M_{j}\right\}$

holds. Then the w-periodic solution of system (1.1) is globally exponentially stable. 
Proof. From Theorem 3.1, we see that system (1.1) has at least one $\omega$-periodic solution $x^{*}(t)=$ $\left(x_{1}^{*}(t), x_{2}^{*}(t), \ldots, x_{n}^{*}(t)\right)^{T}$. Let $x(t)=\left(x_{1}(t), x_{2}(t), \ldots, x_{n}(t)\right)^{T}$ be an arbitrary solution of system (1.1). Let $y(t)=x(t)-x^{*}(t)$, and then we obtain that

$$
\begin{aligned}
y_{i}^{\Delta}(t)= & -c_{i}(t) y_{i}^{\sigma}(t)+\sum_{j=1}^{n} a_{i j}(t)\left[f\left(x_{j}(t)\right)-f\left(x_{j}^{*}(t)\right)\right] \\
& +\sum_{j=1}^{n} b_{i j}(t)\left[f_{j}\left(x_{j}\left(\xi_{i j}(t, x(t))\right)\right)-f_{j}\left(x_{j}^{*}\left(\xi_{i j}(t, x(t))\right)\right)\right], \quad i=1,2, \ldots, n
\end{aligned}
$$

with initial values given by

$$
y_{i}(s)=\varphi_{i}(s)-x_{i}^{*}(s), \quad s \in[-\tau, 0]_{\mathbb{T}}, i=1,2, \ldots, n,
$$

where each $\varphi_{i} \in C\left[(-\tau, 0]_{\mathbb{T}}, \mathbb{R}\right]$ is bounded.

$$
\begin{aligned}
& \text { Let } h(t)=\left(h_{1}(t), h_{2}(t), \ldots, h_{n}(t)\right)^{T} \text { and } \\
& \begin{aligned}
h_{i}(t)= & \sum_{j=1}^{n} a_{i j}(t)\left(f\left(x_{j}(t)\right)-f\left(x_{j}^{*}(t)\right)\right) \\
& +\sum_{j=1}^{n} b_{i j}(t)\left[f_{j}\left(x_{j}\left(\xi_{i j}(t, x(t))\right)\right)-f_{j}\left(x_{j}^{*}\left(\xi_{i j}(t, x(t))\right)\right)\right], \quad i=1,2, \ldots, n .
\end{aligned}
\end{aligned}
$$

From Lemma 4.1, then

$$
y_{i}(t)=e_{\ominus c_{i}}(t, \delta) y_{0}+\int_{\delta}^{t} e_{\ominus c_{i}}(t, s) h_{i}(s) \Delta s, \quad i=1,2, \ldots, n, \delta \in(-\infty, 0]_{\mathbb{T}}, t \geq \delta
$$

is the unique solution of (4.3) with initial value $y(\delta)=y_{0}$. From (4.6), we have

$$
\frac{\left|y_{i}(t)\right|}{e_{\ominus c_{i}}(t, \delta)} \leq\left|y_{0}\right|+\int_{\delta}^{t} \frac{1}{e_{\ominus c_{i}}(s, \delta)}\left|h_{i}(s)\right| \Delta s, \quad i=1,2, \ldots, n, t \geq \delta
$$

and, according to (4.5), we can obtain that

$$
\|h\| \leq\left(\sum_{j=1}^{n} \bar{a}_{i j} M_{j}+\sum_{j=1}^{n} \bar{b}_{i j} M_{j}\right)\|y\| \leq \widehat{M}\|y\|, \quad i=1,2, \ldots, n, t \geq \delta .
$$

Thus, by (4.7), we have

$$
\frac{\|y\|}{e_{\ominus c_{i}}(t, \delta)} \leq\left\|y_{0}\right\|+\int_{\delta}^{t} \widehat{M} \frac{\|y\|}{e_{\ominus c_{i}}(s, \delta)} \Delta s, \quad i=1,2, \ldots, n, t \geq \delta
$$


By virtue of Gronwall's inequality ([32], Corollary 6.7), we obtain

$$
\frac{\|y\|}{e_{\ominus c_{i}}(t, \delta)} \leq\left\|y_{0}\right\| e_{\widehat{M}}(t, \delta), \quad i=1,2, \ldots, n, t \geq \delta
$$

Hence we have

$$
\begin{aligned}
\|y\| & \leq\left\|y_{0}\right\| e_{\widehat{M}}(t, \delta) e_{\ominus c_{i}}(t, \delta) \\
& \leq\left\|y_{0}\right\| e_{\ominus c_{i} \oplus \widehat{M}}(t, \delta) \\
& =\left\|y_{0}\right\| e_{-\left(c_{i}-\widehat{M}\right) /\left(1+\mu c_{i}\right)}(t, \delta) \\
& \leq\left\|y_{0}\right\| e_{-\gamma}(t, \delta), \quad t \geq \delta,
\end{aligned}
$$

where $\gamma=\min _{1 \leq i \leq n}\left\{\left(c_{i}-\widehat{M}\right) /\left(1+\mu c_{i}\right)\right\}, i=1,2, \ldots, n$. We can rewrite (4.10) as follows

$$
\sum_{i=1}^{n}\left\|x_{i}(t)-x_{i}^{*}(t)\right\| \leq\left\|\phi(\delta)-x^{*}(\delta)\right\| e_{-\gamma}(t, \delta), \quad t \geq \delta .
$$

Therefore, the $\omega$-periodic solution of system (1.1) is globally exponentially stable. This completes the proof.

From Theorem 4.2 , when $\mathbb{T}=\mathbb{R}, \xi_{i j}(t, \cdot) \equiv t-\tau_{i j}(t)$, we have the following.

Corollary 4.3. Under assumptions of Theorem 4.2, all the w-periodic solutions of system (1.2) are globally exponentially stable.

When $\mathbb{T}=\mathbb{Z}, \xi_{i j}(n, \cdot) \equiv n-\tau_{i j}(n)$, we have the following.

Corollary 4.4. Under assumptions of Theorem 4.2, all the w-periodic solutions of system (1.3) are globally exponentially stable.

Remark 4.5. Corollaries 3.2 and 4.3 improve the corresponding results obtained in $[4-6,13,14]$ and are different from those in $[8,11,12,16,17]$ and the references cited therein. Therefore, the results of this paper are new, and they complement previously known results. Corollaries 3.3 and 4.4 improve the results obtained in [15].

\section{An Example}

Consider the following cellular neural networks with delays:

$$
x_{i}^{\Delta}(t)=-c_{i}(t) x_{i}(t)+\sum_{j=1}^{2} a_{i j}(t) f_{j}\left(x_{j}(t)\right)+\sum_{j=1}^{2} b_{i j}(t) f_{j}\left(x_{j}\left(t-\tau_{i j}(t)\right)\right)+I_{i}(t),
$$

where $t \in \mathbb{T}, i=1,2, \mathbb{T}$ is a 1 -periodic time scale, $c_{1}(t)=1 / 4+(1 / 8) \sin (2 \pi t), c_{2}(t)=$ $1 / 3+(1 / 6) \cos (2 \pi t), a_{11}(t)=\sin ^{2}(\pi t), a_{12}(t)=a_{21}(t)=(1 / 4) \sin ^{4}(\pi t), a_{22}(t)=|\cos (\pi t)|$, 
$b_{11}(t)=2 \cos ^{2}(\pi t), b_{12}(t)=b_{21}(t)=(1 / 8) \cos ^{6}(\pi t), b_{22}(t)=(1 / 2)|\sin (\pi t)|, f_{1}\left(x_{1}\right)=(1 / 112) x+$ $(1 / 56) \sin \left((1 / 2) x_{1}\right)+3, f_{2}\left(x_{2}\right)=(1 / 126) x+(1 / 42) \cos \left((2 / 3) x_{2}\right)-11, I_{1}(t)=\sin ^{6}(\pi t)$, $I_{2}(t)=3 \cos (2 \pi t), \tau_{11}(t)=e^{\cos (2 \pi t)}, \tau_{12}(t)=3^{\sin ^{2}(\pi t)}, \tau_{21}(t)=\ln \left(\sin ^{4}(\pi t)\right), \tau_{22}(t)=3 / 7+5^{\cos ^{6}(\pi t)}$.

By calculating, we have $\bar{c}_{1}=3 / 8, \underline{c}_{1}=1 / 8, \bar{c}_{2}=1 / 2, \underline{c}_{2}=1 / 6, M_{1}=1 / 56, M_{2}=1 / 42$, $\bar{a}_{11}=1, \bar{a}_{12}=\bar{a}_{21}=1 / 4, \bar{a}_{22}=1, \bar{b}_{11}=2, \bar{b}_{12}=\bar{b}_{21}=1 / 8, \bar{b}_{22}=1 / 2$. It is not difficult to verify that $(\mathrm{H} 1)-(\mathrm{H} 2)$ are satisfied.

Also by calculating, we have that

$$
E=\left(e_{i j}\right)_{2 \times 2}=\left(\begin{array}{cc}
\frac{1}{28} & -\frac{1}{112} \\
-\frac{15}{228} & \frac{1}{28}
\end{array}\right)
$$

is a nonsingular $M$ matrix. If we take $\xi_{1}=\xi_{2}=1$, we can obtain that

$$
-\xi_{1} \underline{c}_{1}+\sum_{j=1}^{2} \xi_{j}\left(\bar{a}_{1 j}+\bar{b}_{1 j}\right) M_{j}=-\frac{7}{112}<0, \quad-\xi_{2} \underline{c}_{2}+\sum_{j=1}^{2} \xi_{j}\left(\bar{a}_{2 j}+\bar{b}_{2 j}\right) M_{j}=-\frac{167}{1344}<0 .
$$

The condition (H3) is satisfied. By Theorems 3.1 and 4.2, we know that system (5.1) has at least one 1-periodic solution, and this solution is globally exponentially stable.

\section{Conclusion}

In this paper, without assuming the boundedness of the activation functions, we establish the stability and existence of periodic solutions of cellular neural networks with delays on time scales. Our results obtained in this paper are completely new even in case of the time scale $\mathbb{T}=\mathbb{R}$ or $\mathbb{Z}$. Besides, our method used in this paper may be used to study other type neural networks such as BAM neural networks, high-order Hopfield neural networks, and Cohen-Grossberg neural networks.

\section{Acknowledgments}

This work is supported by the National Natural Sciences Foundation of People's Republic of China under Grant 10971183, and this work was also supported by IRTSTYN.

\section{References}

[1] L. O. Chua, CNN: A Paradigm for Complexity, World Scientific, Singapore, 1998.

[2] L. O. Chua and L. Yang, "Cellular neural networks: applications," Institute of Electrical and Electronics Engineers, vol. 35, no. 10, pp. 1273-1290, 1988.

[3] T. Roska and J. Vandewalle, Cellular Neural Networks, Wiley, NewYork, NY, USA, 1995.

[4] D. Zhou and J. Cao, "Globally exponential stability conditions for cellular neural networks with timevarying delays," Applied Mathematics and Computation, vol. 131, no. 2-3, pp. 487-496, 2002.

[5] J. Cao, "New results concerning exponential stability and periodic solutions of delayed cellular neural networks," Physics Letters A, vol. 307, no. 2-3, pp. 136-147, 2003.

[6] Y. Li, L. Zhu, and P. Liu, "Existence and stability of periodic solutions of delayed cellular neural networks," Nonlinear Analysis: Real World Applications, vol. 7, no. 2, pp. 225-234, 2006. 
[7] Y. Li and Z. Xing, "Existence and global exponential stability of periodic solution of CNNs with impulses," Chaos, Solitons E Fractals, vol. 33, no. 5, pp. 1686-1693, 2007.

[8] H. Liu and L. Wang, "Globally exponential stability and periodic solutions of CNNs with variable coefficients and variable delays," Chaos, Solitons and Fractals, vol. 29, no. 5, pp. 1137-1141, 2006.

[9] L. Zhou and G. Hu, "Global exponential periodicity and stability of cellular neural networks with variable and distributed delays," Applied Mathematics and Computation, vol. 195, no. 2, pp. 402-411, 2008.

[10] Y. Li, W. Xing, and L. Lu, “Existence and global exponential stability of periodic solution of a class of neural networks with impulses," Chaos, Solitons and Fractals, vol. 27, no. 2, pp. 437-445, 2006.

[11] B. Liu and L. Huang, "Existence and exponential stability of periodic solutions for cellular neural networks with time-varying delays," Physics Letters A, vol. 349, no. 6, pp. 474-483, 2006.

[12] H. Jiang and J. Cao, "Global exponential stability of periodic neural networks with time-varying delays," Neurocomputing, vol. 70, no. 1-3, pp. 343-350, 2006.

[13] J. Cao and Q. Li, "On the exponential stability and periodic solutions of delayed cellular neural networks," Journal of Mathematical Analysis and Applications, vol. 252, no. 1, pp. 50-64, 2000.

[14] Z. Gui and X.-S. Yang, "Stability and existence of periodic solutions of periodic cellular neural networks with time-varying delays," Computers $\mathcal{E}$ Mathematics with Applications, vol. 52, no. 12, pp. 1657-1670, 2006.

[15] Y. Li, "Global stability and existence of periodic solutions of discrete delayed cellular neural networks," Physics Letters A, vol. 333, no. 1-2, pp. 51-61, 2004.

[16] Z. Liu and L. Liao, "Existence and global exponential stability of periodic solution of cellular neural networks with time-varying delays," Journal of Mathematical Analysis and Applications, vol. 290, no. 1, pp. 247-262, 2004.

[17] J. Zhou, Z. Liu, and G. Chen, "Dynamics of periodic delayed neural networks," Neural Networks, vol. 17, no. 1, pp. 87-101, 2004.

[18] F. M. Atici and D. C. Biles, "First order dynamic inclusions on time scales," Journal of Mathematical Analysis and Applications, vol. 292, no. 1, pp. 222-237, 2004.

[19] S. H. Saker, "Oscillation of nonlinear dynamic equations on time scales," Applied Mathematics and Computation, vol. 148, no. 1, pp. 81-91, 2004.

[20] M. Bohner and R. Hilscher, "An eigenvalue problem for linear Hamiltonian dynamic systems," Polytechnica Posnaniensis, no. 35, pp. 35-49, 2005.

[21] Y. Li, X. Chen, and L. Zhao, "Stability and existence of periodic solutions to delayed Cohen-Grossberg BAM neural networks with impulses on time scales," Neurocomputing, vol. 72, no. 7-9, pp. 1621-1630, 2009.

[22] Y. Li and C. Wang, "Uniformly almost periodic functions and almost periodic solutions to dynamic equations on time scales," Abstract and Applied Analysis, vol. 2011, Article ID 341520, 22 pages, 2011.

[23] J. Diblík, M. Růžičková, and Z. Šmarda, "Ważewski's method for systems of dynamic equations on time scales," Nonlinear Analysis:Theory, Methods and Applications, vol. 71, no. 12, pp. e1124-e1131, 2009.

[24] J. Diblík, M. Růžičková, Z. Šmarda, and Z. Šutá, "Asymptotic convergence of the solutions of a dynamic equation on discrete time scales," Abstract and Applied Analysis, vol. 2012, Article ID 580750, 20 pages, 2012.

[25] Y. Li and C. Wang, "Almost periodic solutions of shunting inhibitory cellular neural networks on time scales," Communications in Nonlinear Science and Numerical Simulation, no. 17, pp. 3258-3266, 2012.

[26] J. Diblík and I. Hlavičková, "Combination of Liapunov and retract methods in the investigation of the asymptotic behavior of solutions of systems of discrete equations," Dynamic Systems and Applications, vol. 18, no. 3-4, pp. 507-537, 2009.

[27] J. Diblík, D. Y. Khusainov, I. V. Grytsay, and Z. Šmarda, “Stability of nonlinear autonomous quadratic discrete systems in the critical case," Discrete Dynamics in Nature and Society, vol. 2010, Article ID 539087, 23 pages, 2010.

[28] Y. Li and C. Wang, "Pseudo almost periodic functions and pseudo almost periodic solutions to dynamic equations on time scales," Advances in Difference Equations, vol. 2012, article 77, 2012.

[29] S. Mohamad and K. Gopalsamy, "Dynamics of a class of discrete-time neural networks and their continuous-time counterparts," Mathematics and Computers in Simulation, vol. 53, no. 1-2, pp. 1-39, 2000.

[30] S. Mohamad, "Global exponential stability in continuous-time and discrete-time delayed bidirectional neural networks," Physica D, vol. 159, no. 3-4, pp. 233-251, 2001.

[31] E. R. Kaufmann and Y. N. Raffoul, "Periodic solutions for a neutral nonlinear dynamical equation on a time scale," Journal of Mathematical Analysis and Applications, vol. 319, no. 1, pp. 315-325, 2006. 
[32] M. Bohner and A. Peterson, Dynamic Equations on Time Scales: An Introduction with Applications, Birkhäuser, Boston, Mass, USA, 2001.

[33] M. Bohner, M. Fan, and J. Zhang, "Existence of periodic solutions in predator-prey and competition dynamic systems," Nonlinear Analysis: Real World Applications, vol. 7, no. 5, pp. 1193-1204, 2006.

[34] D. O'Regan, Y. J. Cho, and Y.-Q. Chen, Topological Degree Theory and Applications, vol. 10 of Mathematical Analysis and Applications, Chapman \& Hall/CRC, Boca Raton, Fla, USA, 2006.

[35] A. Berman and R. J. Plemmons, Nonnegative Matrices in the Mathematical Sciences, Academic Press, New York, NY, USA, 1979.

[36] O. Došlý and S. Hilger, "A necessary and sufficient condition for oscillation of the Sturm-Liouville dynamic equation on time scales," Journal of Computational and Applied Mathematics, vol. 141, no. 1-2, pp. 147-158, 2002. 


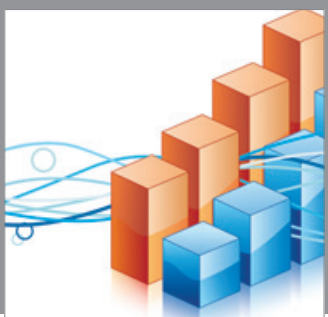

Advances in

Operations Research

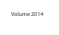

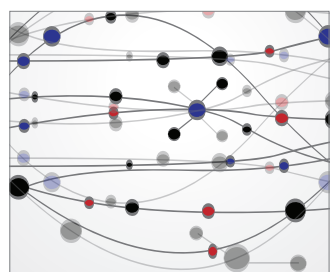

\section{The Scientific} World Journal
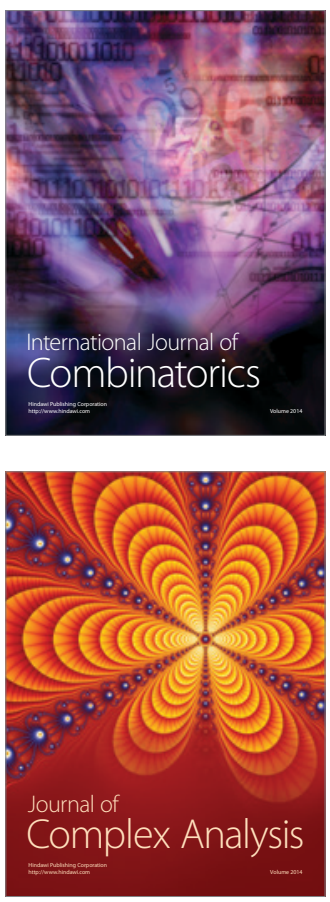

International Journal of

Mathematics and

Mathematical

Sciences
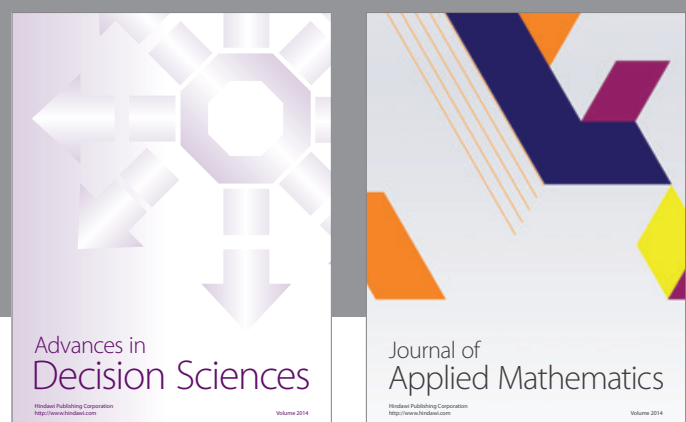

Journal of

Applied Mathematics
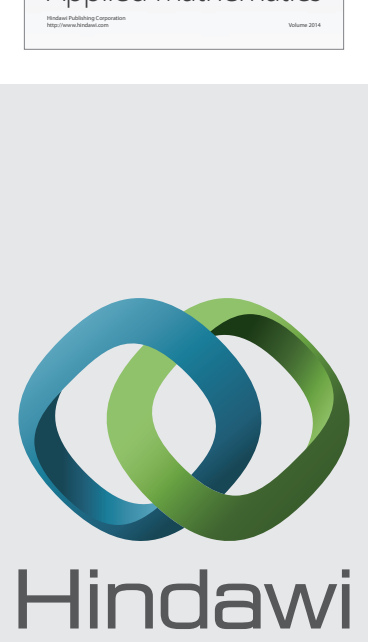

Submit your manuscripts at http://www.hindawi.com
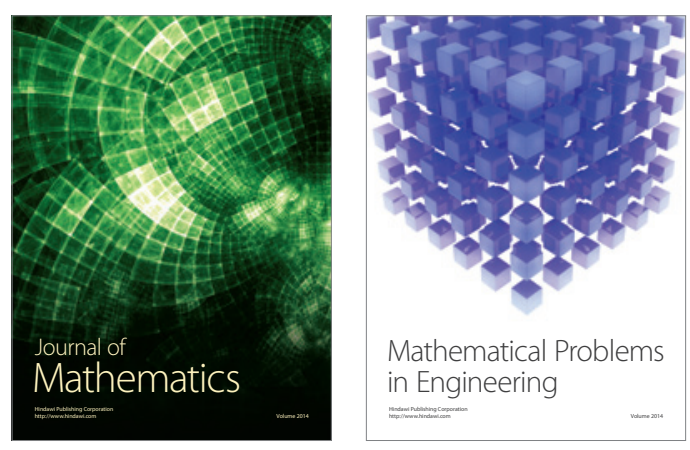

Mathematical Problems in Engineering
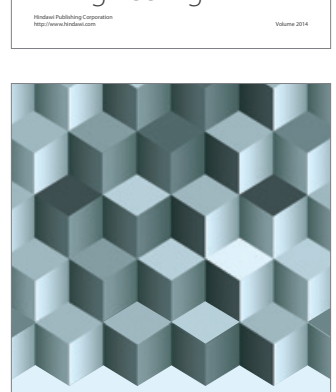

Journal of

Function Spaces
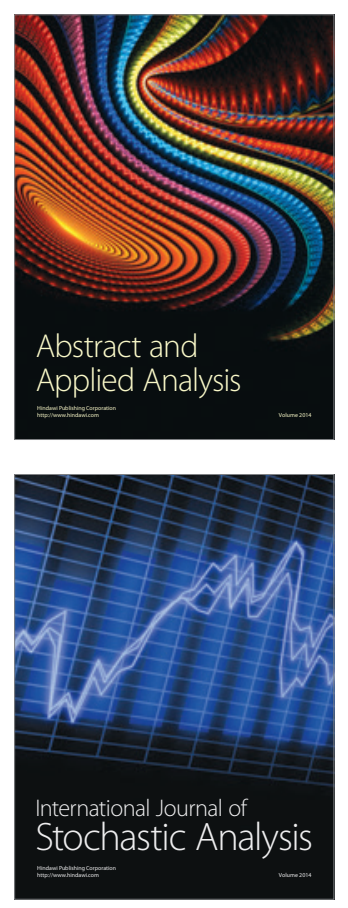

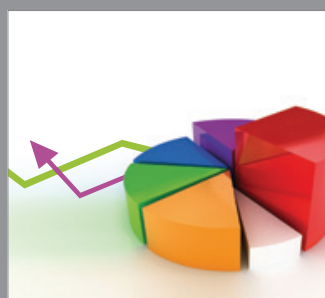

ournal of

Probability and Statistics

Promensencen
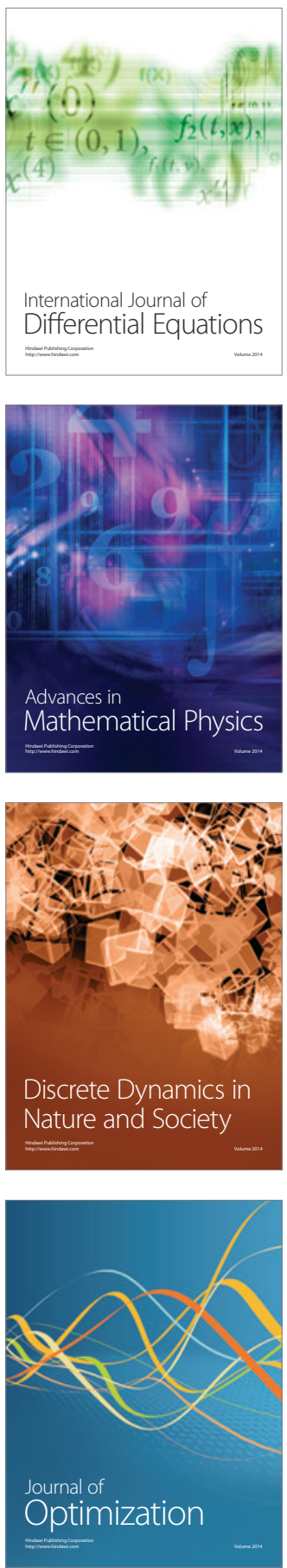